\title{
Quantitative Assessment of Global and Regional Left Ventricular Function With Low-contrast Dose Digital Subtraction Ventriculography*
}

\author{
G. B. John Mancini, M.D.; John McB. Hodgson, M.D.; \\ Victor Legrand, M.D.; Eric R. Bates, M.D.; Fred M. Aueron, M.D.; \\ Michael T. LeFree, B.S.; Joseph S. Smith, M.D.; Glenn J. Beauman, M.S.; \\ and Robert A. Vogel, M.D.
}

Few studies have compared the use of low-contrast dose digital subtraction ventriculography with conventional ventriculography for quantitative assessment of both global and regional left ventricular function. Accordingly, 34 patients underwent conventional ventriculography using $36 \mathrm{ml}$ of ionic contrast material and digital ventriculography (maskmode) using $10 \mathrm{ml}$ of contrast diluted in $10 \mathrm{ml}$ of saline and injected over two seconds. Data from two patients were excluded because of ectopy during cineventriculography and from one because of ectopy during both studies. Enddiastolic and end-systolic volumes were calculated from

D igital intravenous (IV) ventriculography has recently been shown to provide accurate determinations of global and regional left ventricular function compared with conventional contrast ventriculography ${ }^{1.9}$ Although this technique provides highquality ventricular images in a minimally invasive fashion and improved resolution compared with other noninvasive imaging techniques, the use of relatively large doses of contrast material limits its usefulness in certain patient subgroups intolerant to the hemodynamic effects of contrast or in whom repeated ventriculograms are needed. The hemodynamic perturbations induced by contrast material have recently been shown to be similar whether contrast is adminis-

For editorial comment see page 560

tered IV or directly into the left ventricle. ${ }^{10}$ As a consequence, several groups have proposed the use of direct, low-contrast dose left ventriculography to overcome these drawbacks while forgoing the advantage of less invasive IV techniques. Relatively few studies have compared this new method with conventional contrast ventriculography for the assessment of volume and

*From the Division of Cardiology, University of Michigan Medical School, Veterans Administration Medical Center, Ann Arbor.

Supported in part by the Research Service of the Veterans Administration.

Manuscript received August 6; revision accepted September 21.

Reprint requests: Dr. Mancini, VA Medical Center, Ann Arbor 48105 both studies by an area-length method and used to calculate ejection fractions. Regional wall motion was quantitated by the centerline method. Results of linear regression analysis demonstrated high correlations for all parameters (enddiastolic volume, $r=0.85$; end-systolic volume, $r=0.93$; ejection fraction, $r=0.92$; quantitative regional wall motion, $r=0.90$ ). Thus, low-contrast dose digital subtraction ventriculography provides an accurate assessment of both global and regional ventricular function and minimizes the required dose and inherent risks of contrast media.

ejection fraction measurements. ${ }^{6.11 .12}$ What is more, comparative assessment of regional function on a quantitative basis has rarely been reported. ${ }^{13}$ The purpose of this study was to compare standard left ventriculography with low-contrast dose digital ventriculography for the quantitative measurement of both global and regional left ventricular function.

\section{METHODS}

Patient Population

All patients referred for cardiac catheterization were eligible for this study except when renal insufficiency was present. Thirty-four patients, 43 to 67 years of age, were studied. On the basis of coronary arteriography, five were normal and 29 had significant ( $>50$ percent) coronary stenoses of at least one major coronary artery. The majority of patients were taking cardioactive drugs up to the time of cardiac catheterization, but no new medications were administered between the acquisition of the ventriculograms, which were obtained within 15 minutes of each other during a single cardiac catheterization.

\section{Clinical Protocol}

All patients underwent coronary arteriography by the Sones or Judkins technique with multiple angulated views. A 7-French pigtail catheter was advanced into the left ventricle, and a low-contrast dose digital ventriculogram was acquired using $10 \mathrm{ml}$ of sodium meglumine diatrizoate (Renografin 76) diluted and agitated in $10 \mathrm{ml}$ of normal saline solution just prior to power injection at $10 \mathrm{ml} / \mathrm{second}$ for two seconds. This technique caused no significant changes in left ventricular systolic or end-diastolic pressures. Ten to fifteen minutes later, a standard cineventriculogram was acquired using $36 \mathrm{ml}$ of contrast injected over three seconds. All images were obtained in the $30^{\circ}$ right anterior oblique projection. 


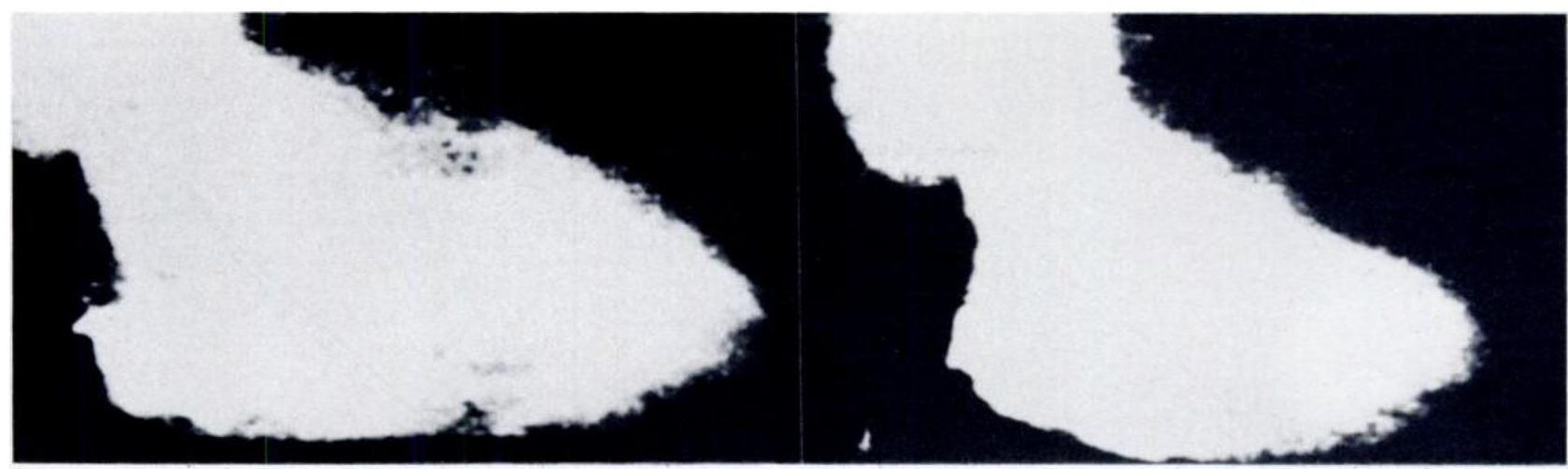

Figure 1. End-diastolic (left) and end-systolic (right) frames of a low-contrast dose, direct digital ventriculogram. Severe inferior hypokinesis is evident.

Imaging System

The angiographic facility utilizes a Philips Optimus M200 unit that provides standard 35-mm cineradiography, as well as ECG-gated, multiple-frame per cardiac cycle, 5-msec/frame exposures onto a 1,000:1 signal-to-noise ratio Plumbicon television camera. Radiation exposure is $25 \mathrm{mcR} /$ frame. For digital ventriculography, the video signals are digitized on-line through a 10-bit $A$ to $D$ converter onto a digital disk with a 7 megabyte/second transfer rate, using a commercial minicomputer (ADAC, DPS-4100C) for final image processing The ventricular images are digitized at 30 frames per second into a $256 \times 256 \times 8$-bit matrix and displayed after digital-to-analogue conversion.

\section{Data Analysis}

The entire digital ventriculographic acquisition was analyzed to obtain precontrast mask frames and the frames constituting the single best opacified beat, excluding ectopic or immediately postectopic beats. In the early phase of the study, the mask was formed by integrating up to $\mathbf{1 6}$ frames precontrast injection, whereas subsequently mask-mode subtraction was performed by matching both precontrast and postcontrast images to the same phase of the cardiac cycle (ECG-synchronized mask-mode subtraction). Because patient motion was kept to a minimum, neither method of mask selection produced significant differences in ventricular quantitation. The processed beat was contrast enhanced to optimize border definition, and the outlines of the largest and smallest ventricular images were drawn and stored (Fig 1). A magnification correction factor was obtained by imaging a $1-\mathrm{cm}$, cross-hatched grid at the same distance as the distance measured from the image intensifier to the midchest level of the subject. End-diastolic and end-systolic volumes and the ejection fraction were then determined by an area-length method. ${ }^{14}$

Regional wall motion was quantitated by the centerline method developed and validated at the University of Washington (Seattle). ${ }^{15.16}$ Briefly, this method measures motion at 100 equidistant chords constructed perpendicular to a centerline drawn midway between the end-diastolic and end-systolic contours. To adjust for heart size, the chord lengths are divided by the perimeter of the enddiastolic contour and expressed in terms of a dimensionless percent shortening fraction. The shortening fraction for each chord is then normalized by subtracting the mean chordal shortening of a normal group and dividing by the SD of chord motion of the normal population. Thus, wall motion is expressed in terms of SDs per chord (SD/C). This standardization allows comparisons between different segments of the same heart and between different hearts. Quantitative wall motion was determined for anterior and inferior regions. Abnormalities of motion in the anterior wall were ascribed to significant stenoses of the left anterior descending or nondominant circumflex systems. Inferior abnormalities were considered to be due to disease in the right coronary or dominant circumflex systems
(Fig 2).

The same methods of volume and wall motion analysis were applied to the cineventriculograms after selection, digitization, and outlining of the largest and smallest endocardial images.

To determine the reproducibility of the measurements, parameters were recalculated from the low dose digital ventriculograms from ten patients by one observer on two ocrasions at least six weeks apart and by a second independent observer. Correlations were determined by linear regression analysis.

\section{Resuits}

Data from two patients who had ectopic beats during cineventriculography and one patient who had ectopy during both conventional and digital ventriculography were excluded.

\section{Volume and Ejection Fraction Measurements}

Figure 3 demonstrates the relationship between end-diastolic volumes determined from the cineventriculograms compared to the digital ventriculograms. The correlation coefficient was 0.85 $(y=0.80 x+27 \mathrm{ml}$, SEE $=20.1 \mathrm{ml}, \mathrm{p}<.001)$. Endsystolic volumes (Fig 4) showed a higher correlation

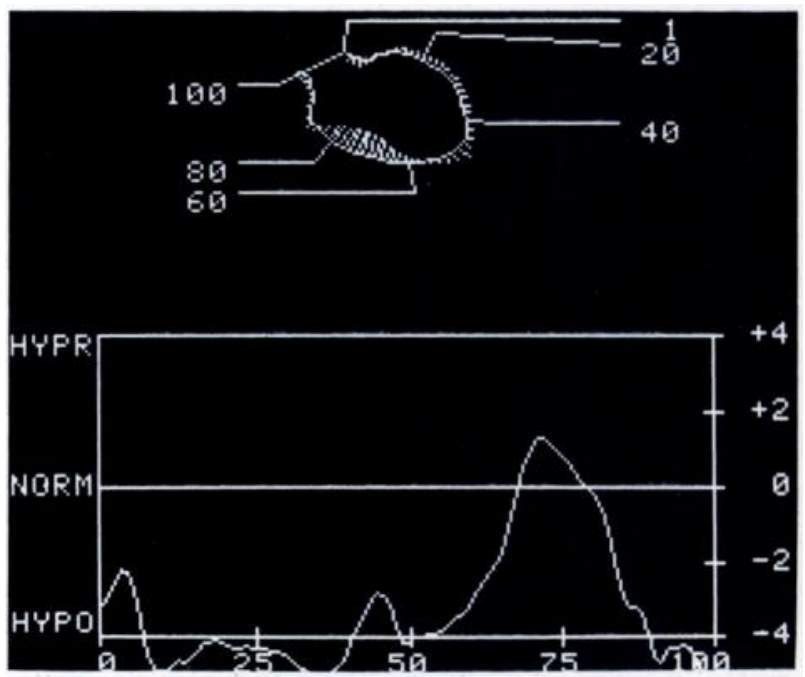

Figure 2. Video display of the quantitative centerline method applied to ventricular outlines (above) from a patient with anterior dyskinesis. Regional wall motion is expressed in SDs per chord and shown in the bottom graph, which has the chord number on the abscissa and the SDs on the ordinate. 


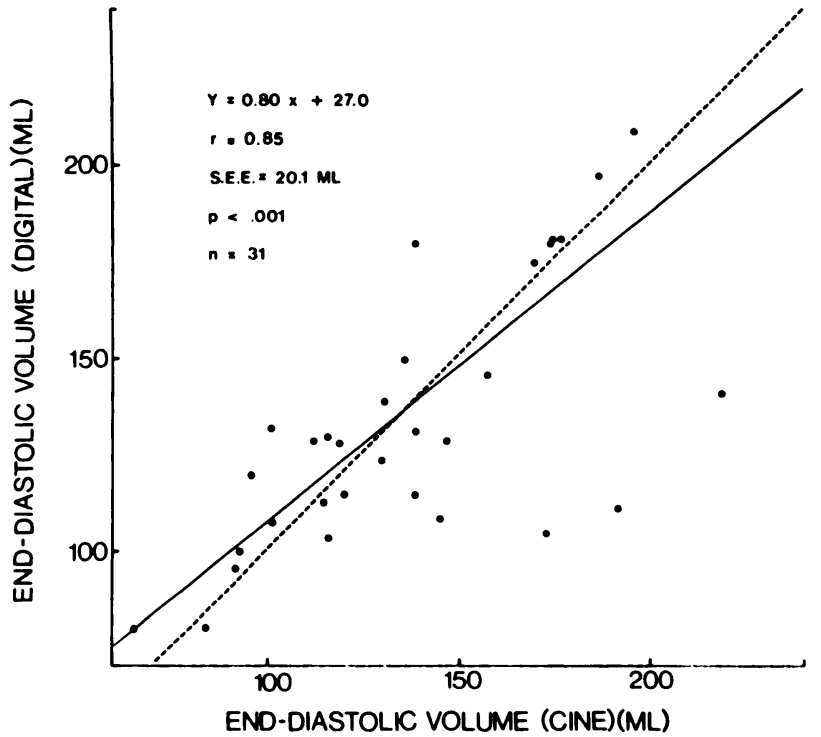

Figurf 3. Relationship between end-diastolic volumes determined by conventional ventriculography (CINE) and low dose, digital ventriculography (DIGITAL). The regression line is solid and the line of identity is dashed. S.E.E. = standard error of the estimate.

$(\mathrm{r}=0.93, \mathrm{y}=0.80 \mathrm{x}+7.9 \mathrm{ml}, \mathrm{SEE}=11.2 \mathrm{ml}, \mathrm{p}<.001)$. The ejection fraction measurements (Fig 5$)$ also displayed a high correlation $(r=0.92, y=0.93 x+6.0$ percent, $\mathrm{SEE}=6.7$ percent, $\mathrm{p}<.001$ ).

\section{Regional Wall Motion}

Figure 6 demonstrates a high degree of correlation for quantitative wall motion assessed from the digital and cineangiographic measurements $(r=.090, y=0.88 x+.02 \mathrm{SD} / \mathrm{C}, \mathrm{SEE}=0.92 \mathrm{SD} / \mathrm{C}, \mathrm{p}<.001)$. No differences were noted in the ability to define either anterior or inferior wall motion.

\section{Reproducibility}

The intraobserver variability was: end-diastolic volume, $\pm 11.7 \mathrm{ml}$ ( \pm 7.4 percent); end-systolic volume, $\pm 8.7 \mathrm{ml}$ ( \pm 12.9 percent);

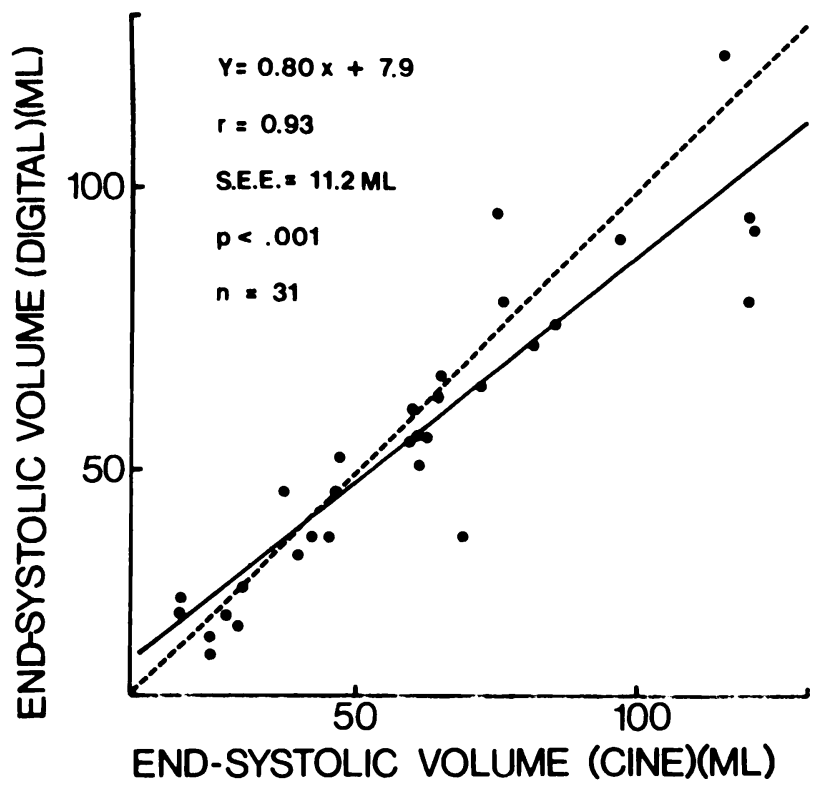

Fisure 4. Results of end-systolic volume correlations. Format and abbreviations as in Figure 3 .

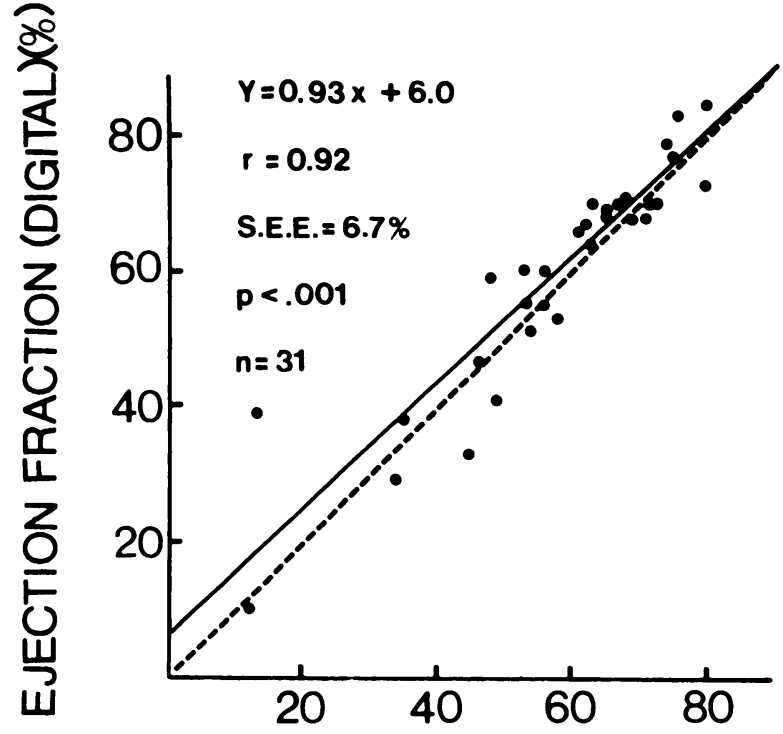

\section{EJECTION FRACTION (CINE)(\%)}

Figure 5. Results of ejection fraction correlations. Format and abbreviations as in Figure 3.

ejection fraction, \pm 6.4 units ( \pm 11.1 percent); regional wall motion, $\pm 0.57 \mathrm{SD} / \mathrm{C}$. The interobserver variability was: end-diastolic volume, $\pm 12.3 \mathrm{ml}$ ( \pm 7 percent); end-systolic volume, $\pm 4.8 \mathrm{ml}$ ( \pm 8.0 percent); ejection fraction, \pm 3.4 units $( \pm 6.0$ percent); regional wall motion, $\pm 0.66 \mathrm{SD} / \mathrm{C}$

\section{Discussion}

The results of this investigation confirm and extend the observations of previous investigators in determining the relative accuracy with which low-contrast dose digital ventriculography can be used to quantitate

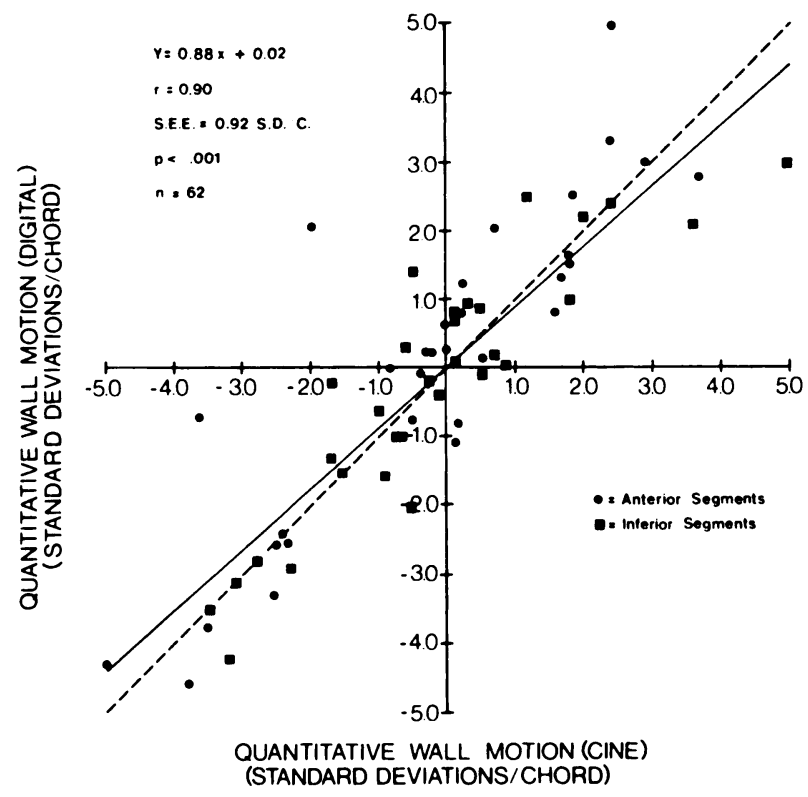

Figure 6. Results of quantitative wall motion correlations. Format and abbreviations as in Figure 3. No differences in quantitation of anterior vs inferior regional motion was noted. Results shown represent pooling of results for anterior and inferior segments from each patient. 
global ventricular function. ${ }^{6.11 \cdot 13}$ The correlation parameters reported herein are intermediate to those previously published. For studies reporting greater correlations, the differences compared with this study that may account for the slightly disparate results include exclusion of patients with abnormal ventricles $^{11}$ and the combining of both end-diastolic and endsystolic volumes to determine only one volume regression. ${ }^{6}$ In a study reporting inferior results for enddiastolic volumes, the relevant difference might be the use of small, undiluted doses of contrast in a study population similar to the current one which contained a substantial proportion of patients with large ventricles and low ejection fractions. ${ }^{12}$ Although not directly examined in this study, empirically it appears that small, undiluted doses of contrast do not consistently provide diagnostic images in patients with large, poorly contracting ventricles or when tachycardia is present. The dilution factor used in this study was based on this observation, and the additional observation that more excessively diluted contrast doses ${ }^{13}$ sometimes also yield inadequate opacification. It should be emphasized that although a rigid protocol is required for investigative studies, the optimal amount, dilution, and rate of contrast injection will be predicated by individual patient characteristics and should be adjusted accordingly as is practiced with standard ventriculography.

The quantitation of ventricular function is facilitated by on-line acquisition and digitization of ventricular images, eliminating the sometimes cumbersome process of separately digitizing cine or video images. In digital form, the images can be easily and routinely analyzed by objective methods. Despite this, little attention has been paid to the quantitation of digital ventricular images for determination of regional wall motion. In large measure, this is due to the absence of a universally accepted method of wall motion analysis, ${ }^{17-19}$ but the need for an objective means of quantitation for correlative studies is underscored by the degree of observer variability when subjective techniques are used. ${ }^{4.20}$ Only one previous study ${ }^{13}$ used a quantitative method to analyze and compare regional function from low dose digital ventriculograms and conventional ventriculograms. A moderate correlation $(r=0.81)$ was shown, which the authors ascribed to a suboptimal image frame rate of only 10/second, not to any potential deficiency of the digital ventriculographic images. Thus, the most significant contribution of the current study is that it extends the limited conclusions of that one prior study regarding the accuracy with which regional wall motion can be assessed from low-contrast dose digital ventriculograms and confirms that with a more appropriate framing rate (30/second), the quantitation of regional wall motion with low dose digital ventriculography correlates highly $(r=0.90)$ with similar quantitation of standard cineventriculograms.

While forgoing the advantage of IV digital ventriculography, which is minimally invasive, low-contrast dose direct ventriculography avoids the hemodynamic perturbations seen with both IV digital ventriculography and conventional ventriculography. ${ }^{10.12 .13}$ This technique may not be as appropriate for screening patients or for outpatient procedures, but it is of definite clinical advantage as an adjunct to cardiac catheterization facilities, particularly when studying patients who do not tolerate the effects of the usual doses of contrast, including those with impaired renal function, aortic stenosis, unstable angina, or congestive failure. In addition, Tobis and co-workers ${ }^{12.21}$ have demonstrated the usefulness of this technique when multiple ventriculograms are needed to assess interventions such as atrial pacing or when biplane imaging is desired but unavailable. This group also demonstrated fewer premature ventricular contractions with the low dose protocol than with conventional ventriculography ( 0 vs 20 percent of patients, respectively). In the current study, this advantage was also evident but less dramatic, in that only three cineangiograms and one digital ventriculogram of 34 could not be analyzed because of excessive ectopy. Even so, when a digital acquisition run demonstrates ectopic beats, subtraction and contrast enhancement frequently still allow diagnostic imaging of faintly opacified sinus beats remote from the ectopic beat, an option not possible with conventional cineangiography.

Low-contrast dose digital subtraction ventriculography can be used accurately to determine both global and regional left ventricular function while minimizing the required dose and inherent risks of contrast media.

ACKNOWLEDGMENTS: The authors are grateful to investigators at the University of Washington, Seattle (Florence H. Sheehan, Edward L. Bolson, and Harold T. Dodge), for providing assistance in implementing the centerline method of wall motion analysis used in this study. The secretarial assistance of Ms. Diane Bauer is also greatly appreciated.

\section{REFERENCES}

1 Mancini GBJ, Norris SL, Peterson KL, Gregoratos G, Widmann T, Ashburn WL, et al. Quantitative assessment of segmental wall motion abnormalities at rest and after atrial pacing using digital intravenous ventriculography. J Am Coll Cardiol 1983; 2:70-76

2 Norris SL, Slutsky RA, Mancini GBJ, Gregoratos G, Ashburn WL, Peterson KL, et al. Comparison of digital intravenous ventriculography with direct left ventriculography for quantitation of left ventricular volumes and ejection fractions. Am J Cardiol 1983; 51:1399-1403

3 Vas R, Diamond GA, Forrester JS, Whiting JS, Swan HJC. Computer enhancement of direct and venous-injected left ventricular contrast angiography. Am Heart J 1981; 102:719-28

4 Vas R, Diamond GA, Forrester JS, Whiting JS, Pfaff MJ, Levisman JA, et al. Computer-enhanced digital angiography: correlation of clinical assessment of left ventricular ejection 
fraction and regional wall motion. Am Heart J 1982; 104:732-9

5 Tobis J, Nalcioglu O, Johnston WD, Seibert A, Iseri LT, Roeck W, et al. Left ventricular imaging with digital subtraction angiography using intravenous contrast injection and fluoroscopic exposure levels. Am Heart J 1982; 104:20-27

6 Kronenberg MW, Price KK, Smith CW, Robertson RM, Perry JM, Pickens DR, et al. Evaluation of left ventricular performance using digital subtraction angiography. Am J Cardiol 1983; 51 : 837-42

7 Goldberg HL, Borer JS, Moses JW. Fisher J, Cohen B, Skelly NT. Digital subtraction intravenous left ventricular intravenous left ventricular angiography: comparison with conventional intraventricular angiography. J Am Coll Cardiol 1983; 1:858-62

8 Nissen SE, Booth D, Waters J, Fassas T, DeMaria AN. Evaluation of left ventricular contractile pattern by intravenous digital subtraction ventriculography: comparison of cineangiography and assessment of interobserver variability. Am J Cardiol 1983; 52:1293-8

9 Engels PHC, Ludwig JW, Verhoeven LAJ. Left ventricular evaluation by digital video subtraction angiography. Radiology $1982 ; 144: 471-4$

10 Mancini GBJ, Ostrander DR, Slutsky RA, Shabetai R, Higgins CB. Comparative effects of ionic contrast agents injected intravenously or directly into the left ventricle: implications for digital angiography. AJR 1983; 140:425-30

11 Sasayama S, Nonogi H, Kawai C, Eiho S, Kuwahara M. Automated method for left ventricular volume measurement by cineventriculography with minimal doses of contrast medium. Am J Cardiol 1981; 48:746-53

12 Tobis JM, Nalcioglu O, Johnston WD, Seibert A, Roeck W, Iseri LT, et al. Correlation of 10-milliliter digital subtraction ventriculograms compared with standard cineangiograms. Am Heart J 1983; 105:946-52

13 Nichols AB, Martin EC, Fles TP, Stugensky KM, Balancio LA, Casarella WJ, et al. Validation of the angiographic accuracy of digital left ventriculography. Am J Cardiol 1983; 51:224-30

14 Sandler H, Dodge HT. The use of single plane angiocardiograms for the calculation of left ventricular volume in man. Am Heart J 1968; 75:325-34

15 Bolson EL, Kilman S, Sheehan FH, Dodge HT. Left ventricular segmental wall motion: a new method using local direction information. IEEE Comput Cardiol 1980; 245-8

16 Sheehan FH, Dodge HT, Mathey DG, Bolson EL, Mitten S. Application of the centerline method: analysis of change in regional left ventricular wall motion in serial studies. IEEE Comput Cardiol 1982; 97-100

17 Gelberg JH, Brundage BH, Glantz S, Parmley WW. Quantitative left ventricular wall motion analysis: a comparison of area, chord and radial methods. Circulation 1979; 59:991-1000

18 Karsch KR, Lamm U, Blanke H, Rentrop KP. Comparison of nineteen quantitative methods for assessment of localized left ventricular wall motion abnormalities. Clin Cardiol 1980; 3:123-8

19 Clayton PD, Jeppson GM, Klausner SC. Should a fixed external reference system be used to analyze left ventricular wall motion. Circulation 1982; 65:1518-21

20 Chaitman BR, DeMoto H, Bristow JD, Fosch J, Rahimtoola SH. Objective and subjective analysis of left ventricular angiograms. Circulation 1975; 52:420-5

21 Tobis J, Nalcioglu O, Johnston WD, Seibert A, Iseri LT, Roeck W, et al. Digital angiography in assessment of ventricular function and wall motion during pacing in patients with coronary artery disease. Am J Cardiol 1983; 51:668-75 